\title{
Selfpolar Norms on an Indefinite Inner Product Space
}

By

\author{
Frank HANSEN*
}

\begin{abstract}
A vector space equipped with an indefinite inner product is investigated. Selfpolar norms on the space are studied and an operator description for quadratic selfpolar norms is developed when the space allows a Hilbert space topology making the indefinite inner product continuous. The selfpolar norms corresponding to a quasi-decomposition of the space are characterised in terms of the operator description and sufficient conditions for topological equivalence are given.
\end{abstract}

\section{§1. Introduction and Preliminaries}

Let $\mathscr{H}$ be a real or complex vector space equipped with a bilinear respective sesquilinear form $\langle\cdot \mid \cdot\rangle$. In the latter case we assume $\langle\cdot \mid \cdot\rangle$ to be conjugate linear in the first variable. If the form $\langle\cdot \mid \cdot\rangle$ is non-degenerate and hermitian we call the pair $(\mathscr{H},\langle\cdot \mid \cdot\rangle)$ an indefinite inner product space.

A norm $p$ on an indefinite inner product space $(\mathscr{H},\langle\cdot \mid \cdot\rangle)$ is said to be selfpolar if

$$
p(x)=\sup _{p(y) \leqq 1}|\langle x \mid y\rangle|, \quad \forall x \in \mathscr{H} .
$$

The concept of a selfpolar norm on an indefinite inner product space is the analogue to the canonical norm

$$
\tau(x)=\langle x \mid x\rangle^{1 / 2}, \quad x \in \mathscr{H},
$$

on a pre-Hilbert space. Indeed, if $\langle\cdot \mid \cdot\rangle$ is positive definite, the norm $\tau$ defined in equation (1.2) is the unique selfpolar norm on $(\mathscr{H},\langle\cdot \mid \cdot\rangle)$. When $\langle\cdot \mid \cdot\rangle$ is indefinite, no canonical norm is available but selfpolar norms can still be found.

Communicated by H. Araki, May 23, 1979.

* Research Institute for Mathematical Sciences, Kyoto University, Kyoto 606, Japan. On leave from Matematisk Institut, Universitetsparken 5, 2100 København $\theta$, Denmark. 
However, the uniqueness disappears and selfpolar norms can be non-equivalent.

In this paper we propose an operator description of quadratic selfpolar norms when $\mathscr{H}$ allows a Hilbert space topology making $\langle\cdot \mid \cdot\rangle$ continuous. Selfpolar norms inducing a quasi-decomposition of $\mathscr{H}$ are studied and characterized in terms of the operator description and sufficient conditions for topological equivalence are given. Finally a number of examples are studied in order to demonstrate the variety of selfpolar norms.

A norm $p$ on an indefinite inner product space $(\mathscr{H},\langle\cdot \mid \cdot\rangle)$ is said to be a partial majorant if the form $\langle\cdot \mid \cdot\rangle$ is separately continuous in the topology induced by $p$ and a majorant if $\langle\cdot \mid \cdot\rangle$ is jointly continuous. It follows from Banach-Steinhaus' Theorem that a complete partial majorant is a majorant.

If $p$ is a partial majorant, then for each $x \in \mathscr{H}$ there is a constant $c(x)>0$ such that

$$
|\langle x \mid y\rangle| \leqq c(x) p(y),
$$

$\forall y \in \mathscr{H}$,

which ensures that the dual norm $p^{\prime}$ defined by

$$
p^{\prime}(x)=\sup _{p(y) \leqq 1}|\langle x \mid y\rangle|, \quad x \in \mathscr{H},
$$

is finite everywhere.

Let $p_{1}$ and $p_{2}$ be partial majorants and $k$ a positive constant. From the definition (1.4) it easily follows that

$$
\begin{aligned}
& p_{1} \leqq k p_{2} \Rightarrow p_{2}^{\prime} \leqq k p_{1}^{\prime}, \\
& (k p)^{\prime}=k^{-1} p^{\prime} .
\end{aligned}
$$

If $p$ is a majorant, there is a constant $c>0$ such that

$$
|\langle x \mid y\rangle| \leqq c p(x) p(y),
$$

$$
\forall x, y \in \mathscr{H},
$$

and it follows that $p^{\prime} \leqq c p$, thus the topology induced by the dual norm $p^{\prime}$ is weaker than the topology induced by $p$.

A partial majorant $p$ is selfpolar if and only if $p^{\prime}=p$. If $p$ is selfpolar we obtain

$$
|\langle x \mid y\rangle| \leqq p(x) p(y), \quad \forall x, y \in \mathscr{H},
$$

thus $p$ is a majorant. Furthermore $p$ is minimal among norms satisfying (1.7). If conversely $p$ is a majorant and therefore satisfies (1.6) for some constant $c>0$, Aronszajn has shown [1], that there exists a selfpolar norm $p_{s}$ such that

$$
p_{s} \leqq \sqrt{c} p .
$$


Though $p_{s}$ is minimal under (1.7) it is not unique.

We will briefly mention Aronszajn's procedure. Possibly by multiplying the majorant $p$ with a constant we can assume that $p$ satisfies (1.7), that is $p^{\prime} \leqq p$. We put

$$
p_{1}(x)=\sqrt{\frac{P(x)^{2}+p^{\prime}(x)^{2}}{2}}, \quad x \in \mathscr{H}
$$

and observe that

$$
p_{1} \leqq p
$$

and

$$
|\langle x \mid y\rangle| \leqq \frac{p(x) p^{\prime}(y)+p^{\prime}(x) p(y)}{2} \leqq p_{1}(x) p_{1}(y), \quad \forall x, y \in \mathscr{H},
$$

where we have used the definition of $p^{\prime}$, the hermicity of $\langle\cdot \mid \cdot\rangle$ and CauchySchwartz inequality on $\boldsymbol{R}^{2}$. Iterating this procedure we get a sequence of norms $\left(p_{n}\right)_{n \in N}$ satisfying

$$
\begin{array}{lr}
p_{n+1} \leqq p_{n} \leqq p, & \forall n \in \mathbb{N}, \\
|\langle x \mid y\rangle| \leqq p_{n}(x) p_{n}(y), & \forall x, y \in \mathscr{H}, \forall n \in \mathbb{N} .
\end{array}
$$

Since $\left(p_{n}\right)_{n \in N}$ is decreasing the definition

$$
p_{s}(x)=\inf _{n \in N} p_{n}(x) \leqq p(x),
$$

$x \in \mathscr{H}$,

gives a seminorm $p_{s}$ satisfying

$$
|\langle x \mid y\rangle| \leqq p_{s}(x) p_{s}(y),
$$

$\forall x, y \in \mathscr{H}$,

and since $\langle\cdot \mid \cdot\rangle$ is non-degenerate $p_{s}$ is actually a norm. It is not difficult to verify that $p_{s}$ is selfpolar. For a detailed discussion we refer to Lemma IV. 4.1 in [3].

Suppose finally that the inner product $\langle\cdot \mid \cdot\rangle$ is definite. The definition

$$
\tau(x)=|\langle x \mid x\rangle|^{1 / 2},
$$

$x \in \mathscr{H}$,

makes $\tau$ a norm which by Cauchy-Schwartz inequality satisfies

$$
|\langle x \mid y\rangle| \leqq \tau(x) \tau(y),
$$

$\forall x, y \in \mathscr{H}$,

that is $\tau^{\prime} \leqq \tau$. But for each $x \in \mathscr{H}, x \neq 0$

$$
\tau^{\prime}(x)=\sup _{\tau(y) \leqq 1}|\langle x \mid y\rangle| \geqq\left|\left\langle x \mid \frac{x}{\tau(x)}\right\rangle\right|=\tau(x)
$$

thus $\tau$ is selfpolar. If now $p$ also is a selfpolar norm, then $\tau(x)=|\langle x \mid x\rangle|^{1 \text { i }}$ 
$\leqq p(x), x \in \mathscr{H}$ and by (1.5) $p=p^{\prime} \leqq \tau^{\prime}=\tau$ thus $p=\tau$

\section{§2. Selfpolar Quadratic Norms}

A norm $p$ on a vector space $\mathscr{H}$ is said to be quadratic if there is a positive definite inner product $(\cdot \mid \cdot)_{p}$ on $\mathscr{H}$ such that

$$
p(x)=(x \mid x)_{p}^{1 / 2}, \quad \forall x \in \mathscr{H} .
$$

Lemma 2.1. Let $(\mathscr{H},\langle\cdot \mid \cdot\rangle)$ be an indefinite inner product space and $p$ a quadratic norm on $\mathscr{H}$ satisfying

$$
|\langle x \mid y\rangle| \leqq p(x) p(y),
$$$$
\forall x, y \in \mathscr{H}
$$

The selfpolar norm $p_{s} \leqq p$ constructed in the introduction is quadratic.

Proof. Let $(\overline{\mathscr{H}}, p)$ denote the $p$-completion of $\mathscr{H}$. The form $\langle\cdot \mid \cdot\rangle$ extends in view of (2.2) to a (possibly degenerate) hermitian, jointly continuous form on $(\overline{\mathscr{H}}, p)$ which implies the existence of a bounded selfadjoint operator $Q$ on $(\overline{\mathscr{H}}, p)$ such that

$$
\langle x \mid y\rangle=(x \mid Q y)_{p}, \quad \forall x, y \in(\overline{\mathscr{H}}, p) .
$$

The dual norm $p^{\prime}$ satisfies

$$
p^{\prime}(x)=p(Q x)=\left(x \mid Q^{2} x\right)_{p}^{1 / 2}, \quad \forall x \in \mathscr{H},
$$

and is thus quadratic because the form $\left(x \mid Q^{2} y\right)_{p}$ is positive semi-definite on $(\overline{\mathscr{H}}, p)$ and (2.4) implies that it is positive definite on $\mathscr{H}$. The norm $p_{1}$ defined in (1.9) is now easily shown to be quadratic and by iteration we get that each norm in the sequence $\left(p_{n}\right)_{n \in \boldsymbol{N}}$ is quadratic. It follows that $p_{s}$ defined in (1.14) satisfies the parallelogram identity and is thus by a standard argument quadratic.

Q.E.D.

Let $(\mathscr{H},\langle\cdot \mid \cdot\rangle)$ be an indefinite inner product space and suppose $\mathscr{H}$ is equipped with a positive definite inner product $(\cdot \mid \cdot)$ with corresponding norm

$$
\|x\|=(x \mid x)^{1 / 2},
$$

$x \in \mathscr{H}$

making $\mathscr{H}$ a Hilbert space. We furthermore assume that the indefinite inner product $\langle\cdot \mid \cdot\rangle$ is jointly continuous in the Hilbert space topology, that is $\|\cdot\|$ is a majorant. This implies the existence of a bounded selfadjoint operator $\eta$ on $(\mathscr{H},(\cdot \mid \cdot))$ such that 


$$
\langle x \mid y\rangle=(x \mid \eta y),
$$

$\forall x, y \in \mathscr{H}$.

Since $\langle\cdot \mid \cdot\rangle$ is non-degenerate 0 is not an eigenvalue for $\eta$. We will use the notion of orthogonality relative to the indefinite inner product $\langle\cdot \mid \cdot\rangle$ and write

$$
x \perp y \quad \text { if }\langle x \mid y\rangle=0, \quad x, y \in \mathscr{H} .
$$

Proposition 2.2. Let $p$ be a selfpolar norm on $(\mathscr{H},\langle\cdot \mid \cdot\rangle)$. There is a constant $c>0$ such that

$$
\frac{1}{c}\|\eta x\| \leqq p(x) \leqq c\|x\|, \quad \forall x \in \mathscr{H} .
$$

Proof. Let $(\overline{\mathscr{H}}, p)$ denote the $p$-completion of $\mathscr{H}$. Since $\langle\cdot \mid \cdot\rangle$ is jointly $p$-continuous $\langle\cdot \mid \cdot\rangle$ extends to a bilinear (respectively sesquilinear) hermitian form on $(\overline{\mathscr{H}}, p)$ satisfying

$$
|\langle x \mid y\rangle| \leqq p(x) p(y), \quad \forall x, y \in(\overline{\mathscr{H}}, p) .
$$

The seminorm $p^{\prime}$ defined by

$$
p^{\prime}(x)=\sup _{p(y) \leqq 1}|\langle x \mid y\rangle|, \quad x \in(\overline{\mathscr{H}}, p),
$$

is continuous in the $p$-topology $\left(p^{\prime} \leqq p\right)$ and since $p^{\prime}$ coincides with $p$ on the dense subset $\mathscr{H}$ we conclude that

$$
p^{\prime}(x)=p(x), \quad \forall x \in(\overline{\mathscr{H}}, p) .
$$

Especially we get that $\langle\cdot \mid \cdot\rangle$ is non-degenerate on $(\overline{\mathscr{H}}, p)$. Let now $\left(x_{n}\right)_{n \in N}$ be a sequence in $\mathscr{H}$ for which $x_{n} \rightarrow 0$ and $x_{n}{ }^{p} \rightarrow x \in(\overline{\mathscr{H}}, p)$, then

$$
\langle x \mid y\rangle=\lim \left\langle x_{n} \mid y\right\rangle=0, \quad \forall y \in \mathscr{H} .
$$

As $\langle\cdot \mid \cdot\rangle$ is non-degenerate on $(\overline{\mathscr{H}}, p)$ and $\mathscr{H}$ is dense we conclude that $x=0$. The injection mapping $i: \mathscr{H} \rightarrow(\overline{\mathscr{H}}, p)$ is thus a closable linear mapping with a complete domain, therefore continuous according to Banach's theorem. This proves the existence of a constant $c>0$ for which $p(x) \leqq c\|x\|, \forall x \in \mathscr{H}$. Using that $p$ is selfpolar we get

$$
\begin{aligned}
p(x) & =\sup _{p(y) \leqq 1, y \in \mathscr{P}}|\langle x \mid y\rangle|=\sup _{p(y) \leqq 1, y \in \mathscr{P}}|(x \mid \eta y)| \\
& \geqq \sup _{c\|y\| \leqq 1}|(\eta x \mid y)|=\frac{1}{c}\|\eta x\|, \quad \forall x \in \mathscr{H} .
\end{aligned}
$$


Since we can always find selfpolar norms on $(\mathscr{H},\langle\cdot \mid \cdot\rangle)$ in the above situation, for example by using the procedure described in Section 1 on the majorant $\|\cdot\|$, we obtain the following:

Corollary 2.3. If 0 is not in the spectrum of $\eta$ then there is exactly one equivalence class of selfpolar norms. This class contains quadratic norms and the norms in the class are equivalent to $\|\cdot\|$.

A simple and important way of constructing quadratic selfpolar norms is provided by the following lemma.

Lemma 2.4. Let $P^{+}$and $P^{-}$be the spectral projections of $\eta$ corresponding to the positive respective negative part of the spectrum and put

$$
p(x)=\sqrt{\left\langle P^{+} x \mid P^{+} x\right\rangle-\left\langle P^{-} x \mid P^{-} x\right\rangle}, \quad x \in \mathscr{H} .
$$

Then $p$ is a quadratic selfpolar norm.

Proof. The form

$$
\begin{aligned}
(x \mid y)_{p} & =\left\langle P^{+} x \mid P^{+} y\right\rangle-\left\langle P^{-} x \mid P^{-} y\right\rangle \\
& =(x|| \eta \mid y),
\end{aligned}
$$$$
x, y \in \mathscr{H},
$$

is a positive definite inner product, thus $p$ is a quadratic norm. Furthermore

$$
\begin{array}{rlr}
\langle x \mid y\rangle & =(x \mid \eta y)=\left(P^{+} x \mid \eta P^{+} y\right)+\left(P^{-} x \mid \eta P^{-} y\right) \\
& =\left\langle P^{+} x \mid P^{+} y\right\rangle+\left\langle P^{-} x \mid P^{-} y\right\rangle, & \forall x, y \in \mathscr{H},
\end{array}
$$

which by Cauchy-Schwartz inequality first applied on the definite subspaces $P^{+} \mathscr{H}$ and $P^{-} \mathscr{H}$ and then on $\boldsymbol{R}^{2}$ gives

$$
\begin{aligned}
& |\langle x \mid y\rangle| \leqq\left|\left\langle P^{+} x \mid P^{+} y\right\rangle\right|+\left|\left\langle P^{-} x \mid P^{-} y\right\rangle\right| \\
& \leqq\left\langle P^{+} x \mid P^{+} x\right\rangle^{1 / 2} \cdot\left\langle P^{+} y \mid P^{+} y\right\rangle^{1 / 2}+\left|\left\langle P^{-} x \mid P^{-} x\right\rangle\right|^{1 / 2} \cdot\left|\left\langle P^{-} y \mid P^{-} y\right\rangle\right|^{1 / 2} \\
& \leqq p(x) p(y), \quad \forall x, y \in \mathscr{H} .
\end{aligned}
$$

This shows that $p^{\prime} \leqq p$. Now for $x \neq 0$ we put

$$
\hat{x}=\frac{1}{p(x)}\left(P^{+} x-P^{-} x\right)
$$

and observe that $p(\hat{x})=1$ and $\langle x \mid \hat{x}\rangle=p(x)$.

Q.E.D.

Taking different Hilbert space majorants $\|\cdot\|$ and thus varying $\eta$, we can construct by applying Lemma 2.4 a family of quadratic selfpolar norms, which are different if $\langle\cdot \mid \cdot\rangle$ is indefinite. However, these norms are all equivalent as will be shown in Proposition 4.1. 
Theorem 2.5. A norm $p$ on $\mathscr{H}$ is quadratic and selfpolar if and only if there is a positive, bounded operator $T$ on $(\mathscr{H},(\cdot \mid \cdot))$ such that

(i) 0 is not an eigenvalue for $T$,

(ii) $\eta \mathscr{H} \subset D\left(T^{-1 / 2}\right)$,

(iii) $\left\|T^{1 / 2} x\right\|=\left\|T^{-1 / 2} \eta x\right\|$, $\forall x \in \mathscr{H}$

(iv) $p(x)=\left\|T^{1 / 2} x\right\|$, $\forall x \in \mathscr{H}$.

The operator $T$ is unique.

Proof. Let $p$ be a quadratic selfpolar norm on $\mathscr{H}$. By assumption $p$ is of the form $p(x)=(x \mid x)_{p}^{1 / 2}, x \in \mathscr{H}$ where $(\cdot \mid \cdot)_{p}$ is a positive definite inner product on $\mathscr{H}$. In view of Proposition 2.2 there is a constant $c>0$ such that $p \leqq c\|\cdot\|$, hence

$$
\left|(x \mid y)_{p}\right| \leqq p(x) p(y) \leqq c^{2}\|x\| \cdot\|y\|, \quad \forall x, y \in \mathscr{H},
$$

where we have used Cauchy-Schwartz inequality on $(\cdot \mid \cdot)_{p}$. We conclude that $(\cdot \mid \cdot)_{p}$ is of the form

$$
(x \mid y)_{p}=(x \mid T y), \quad \forall x, y \in \mathscr{H},
$$

where $T$ is a positive, bounded operator on $(\mathscr{H},(\cdot \mid \cdot))$. Consequently

$$
p(x)=\left\|T^{1 / 2} x\right\|,
$$

$\forall x \in \mathscr{H}$.

Since $p$ is a norm $T$ does not have 0 as an eigenvalue. The unicity of a positive $T$ satisfying (2.20) follows from the polarisation identity. The mapping

$$
\begin{aligned}
D\left(T^{-1 / 2}\right) \ni y & \rightarrow\left|\left(T^{-1 / 2} y \mid \eta x\right)\right| \\
& =\left|\left\langle T^{-1 / 2} y \mid x\right\rangle\right| \\
& \leqq p\left(T^{-1 / 2} y\right) p(x) \\
& =\|y\| p(x),
\end{aligned}
$$$$
x \in \mathscr{H},
$$

is continuous which shows that $\eta \mathscr{H} \subset D\left(\left(T^{-1 / 2}\right)^{*}\right)=D\left(T^{-1 / 2}\right)$. Furthermore

$$
\begin{aligned}
\left\|T^{1 / 2} x\right\| & =p(x)=\sup _{p(y) \leqq 1}|\langle x \mid y\rangle| \\
& =\sup _{\left\|T^{1 / 2} y\right\| \leqq 1}\left|\left(T^{-1 / 2} \eta x \mid T^{1 / 2} y\right)\right| \\
& =\left\|T^{-1 / 2} \eta x\right\|,
\end{aligned}
$$

where we have used that $R\left(T^{1 / 2}\right)$ is dense in $\mathscr{H}$.

If conversely $T$ is a positive, bounded operator satisfying (i), (ii), (iii) and (iv), then $p$ is quadratic and since the range $R\left(T^{1 / 2}\right)$ is dense in $\mathscr{H}$ we have 


$$
\begin{aligned}
p^{\prime}(x) & =\sup _{p(y) \leqq 1}|\langle x \mid y\rangle|=\sup _{p(y) \leqq 1}|(x \mid \eta y)| \\
& =\sup _{\left\|T^{1 / 2} y\right\| \leqq 1}\left|\left(T^{-1 / 2} \eta x \mid T^{1 / 2} y\right)\right|=\left\|T^{-1 / 2} \eta x\right\| \\
& =p(x), \quad \forall x \in \mathscr{H} .
\end{aligned}
$$

Proposition 2.6. Let $p$ be a quadratic selfpolar norm on $\mathscr{H}$ and $T$ the operator of Theorem 2.5. Then

(i) $T^{-1 / 2} \eta \mathscr{H}$ is dense in $\mathscr{H}$,

(ii) there is a unique selfadjoint unitary $S$ on $(\mathscr{H},(\cdot \mid \cdot))$ such that $S T^{-1 / 2} \eta=T^{1 / 2}$,

(iii) $\eta \mathscr{H}$ is a core for $T^{-1 / 2}$.

Proof. According to Theorem 2.5, (iii) there is an isometry $S: \overline{R\left(T^{-1 / 2} \eta\right)}$ $\rightarrow \overline{R\left(T^{1 / 2}\right)}=\mathscr{H}$ such that

$$
S T^{-1 / 2} \eta x=T^{1 / 2} x, \quad \forall x \in \mathscr{H} .
$$

We extend $S$ to a co-isometry on $(\mathscr{H},(\cdot \mid \cdot))$ with support $\overline{R\left(T^{-1 / 2} \eta\right)}$ and observe that

$$
T^{-1 / 2} \eta=S^{*} S T^{-1 / 2} \eta=S^{*} T^{1 / 2} .
$$

We have $S^{*} x=T^{-1 / 2} \eta T^{-1 / 2} x$ for $x \in D\left(T^{-1 / 2}\right)$ which shows that $S^{*}$ is symmetric, thus selfadjoint. In particular $R\left(T^{-1 / 2} \eta\right)$ is dense in $\mathscr{H}$ and $S$ is a unitary. Let $x \in D\left(T^{-1 / 2}\right)$ and choose a sequence $\left(y_{n}\right)_{n \in \boldsymbol{N}}$ in $\mathscr{H}$ such that $T^{-1 / 2} \eta y_{n} \rightarrow T^{-1 / 2} x$. Since $T^{1 / 2}$ is continuous $\eta y_{n} \rightarrow x$ which proves (iii).

Q.E.D.

Lemma 2.7. Let $T_{1}$ and $T_{2}$ be positive, bounded operators on $(\mathscr{H},(\cdot \mid \cdot))$ which do not have 0 as an eigenvalue and let $p_{1}$ and $p_{2}$ be the norms defined by

$$
p_{i}(x)=\left\|T_{i} x\right\|, \quad x \in \mathscr{H}, \quad i=1,2 .
$$

Then $p_{1}$ and $p_{2}$ are equivalent if and only if $R\left(T_{1}\right)=R\left(T_{2}\right)$.

Proof. Let $T$ be a positive, bounded operator on $(\mathscr{H},(\cdot \mid \cdot))$ which does not have 0 as an eigenvalue and let $p$ be the norm defined by $p(x)=\|T x\|, x \in \mathscr{H}$. We first show that $x \in R(T)$ if and only if there is a constant $c \geqq 0$ such that

$$
|(x \mid y)| \leqq c p(y), \quad \forall y \in \mathscr{H} .
$$

Indeed, since $T^{-1}$ is selfadjoint, $x \in D\left(T^{-1}\right)=R(T)$ if and only if the map $D\left(T^{-1}\right)$ $\ni z \rightarrow\left(T^{-1} z \mid x\right)$ is continuous. Putting $z=T y, y \in \mathscr{H}$ we get that $x \in D\left(T^{-1}\right)$ if and only if the map $T y, y \in \mathscr{H} \rightarrow(y \mid x)$ is continuous. 
This applied on $p_{1}$ and $p_{2}$ shows that $R\left(T_{1}\right)=R\left(T_{2}\right)$ if $p_{1}$ and $p_{2}$ are equivalent. Suppose now $R\left(T_{1}\right)=R\left(T_{2}\right)$ and define

$$
V_{12}=T_{1}^{-1} T_{2} \text {. }
$$

Let $x_{n} \rightarrow 0$ and assume $V_{12} x_{n} \rightarrow y$. Since $T_{2} x_{n} \rightarrow 0$ and $T_{1}^{-1}$ is closed we get $y=0$, thus $V_{12}$ is closable. The assumption yields that the domain of $V_{12}$ is the whole space $\mathscr{H}$, thus $V_{12}$ is bounded. Since $T_{1}^{-1}$ and $T_{2}$ are selfadjoint we get that

$$
V_{12}^{*} x=T_{2} T_{1}^{-1} x, \quad \forall x \in R\left(T_{1}\right)=R\left(T_{2}\right) .
$$

Furthermore

$$
\begin{aligned}
& p_{2}(y)=\left\|T_{2} y\right\|=\left\|V_{12}^{*} T_{1} y\right\| \\
& \leqq\left\|V_{12}\right\| p_{1}(y), \\
& \forall y \in \mathscr{H} .
\end{aligned}
$$

Similarly we get that $V_{21}=T_{2}^{-1} T_{1}$ is bounded and that $p_{1}(y) \leqq\left\|V_{21}\right\| p_{2}(y)$, $\forall y \in \mathscr{H}$.

Corollary 2.8. Let $p_{1}$ and $p_{2}$ be quadratic and selfpolar norms on $\mathscr{H}$ and let $T_{1}$ and $T_{2}$ be the corresponding operators on $(\mathscr{H},(\cdot \mid \cdot))$ defined in Theorem 2.5. Then $p_{1}$ and $p_{2}$ are equivalent if and only if $R\left(T_{1}^{1 / 2}\right)=R\left(T_{2}^{1 / 2}\right)$.

Example 2.9. Let $(\mathscr{H},\langle\cdot \mid \cdot\rangle)$ be the real Hilbert space $\boldsymbol{R}^{2}$ equipped with the indefinite inner product

$$
\langle x \mid y\rangle=x_{1} y_{1}-x_{2} y_{2},
$$

$x, y \in \mathscr{H}$,

and let $p$ be the norm on $\mathscr{H}$ with its unit ball as described in Figure 1 . It is not

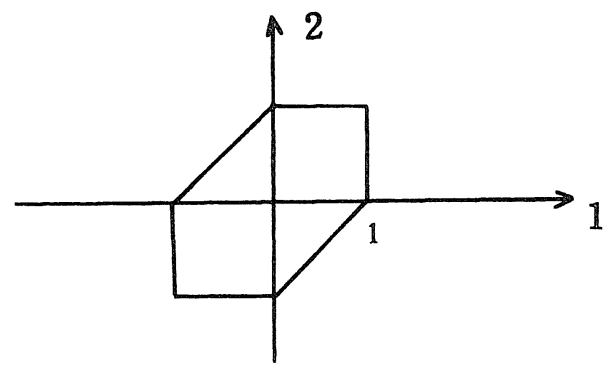

Figure 1

difficult to verify that $p$ is selfpolar, but $p$ is not quadratic.

Example 2.10. Let $\mathscr{H}$ be the real Hilbert space $l^{2}(\mathbb{N}, \mu)$, where $\mu$ is the measure defined by $\mu(n)=n^{2}, n \in N$. It follows from Cauchy-Schwartz inequality that $\mathscr{H}$ is a subspace of $l^{1}(\mathbb{N})$ and that 


$$
\|x\|_{1} \leqq c\|x\|_{\mu}
$$

$\forall x \in \mathscr{H}$,

where the best possible constant $c=\left(\sum_{n=1}^{\infty} n^{-2}\right)^{1 / 2}=\pi / \sqrt{6}$.

Let for each $j \in \boldsymbol{N},(j)_{m}=0,1$, be the coefficients in the binary expansion

$$
j=\sum_{m=0}^{\infty}(j)_{m} 2^{m}
$$

of $j$ and define

$$
\begin{aligned}
& \varepsilon_{i j}=(-1)^{(j)_{i}}, \quad \text { for } i \leqq j, \text { and } \\
& \varepsilon_{i j}=\varepsilon_{j i}, \quad \text { for } i \geqq j, \quad i, j \in \mathbf{N} \text {. }
\end{aligned}
$$

We put

$$
\langle x \mid y\rangle=\sum_{i, j=1}^{\infty} \varepsilon_{i j} x_{i} y_{j}, \quad x, y \in \mathscr{H},
$$

and observe either directly or by passing to equation $(2.40)$ that $\langle\cdot \mid \cdot\rangle$ is a non-degenerate hermitian bilinear form on $\mathscr{H}$ such that

$$
|\langle x \mid y\rangle| \leqq\|x\|_{1}\|y\|_{1} \leqq c^{2}\|x\|_{\mu}\|y\|_{\mu}, \quad \forall x, y \in \mathscr{H} .
$$

Hence $(\mathscr{H},\langle\cdot \mid \cdot\rangle)$ is an indefinite inner product space which allows a Hilbert space majorant $\|\cdot\|_{\mu}$. We will prove that $\|\cdot\|_{1}$ is selfpolar on $(\mathscr{H},\langle\cdot \mid \cdot\rangle)$. Equation (2.36) tells that $\|\cdot\|_{1}^{\prime} \leqq\|\cdot\|_{1}$. Take now $x \in \mathscr{H}$ and $\varepsilon>0$. There is an $n_{0} \in \boldsymbol{N}$ such that

$$
\sum_{n>n_{0}}\left|x_{n}\right|<\frac{\varepsilon}{2}
$$

and a $j_{0} \geqq n_{0}$ such that

$$
\varepsilon_{i j_{0}}=\operatorname{sign} x_{i}, \text { whenever } x_{i} \neq 0, \text { for } i=1, \ldots, n_{0} .
$$

We take $y=\left(y_{n}\right)_{n \in \boldsymbol{N}}$ with

$$
y_{n}= \begin{cases}1, & n=j_{0}, \\ 0, & n \neq j_{0}\end{cases}
$$

and observe that $y \in \mathscr{H},\|y\|_{1}=1$ and

$$
\begin{aligned}
\langle x \mid y\rangle & =\sum_{i, j=1}^{\infty} \varepsilon_{i j} x_{i} y_{j}=\sum_{i=1}^{\infty} \varepsilon_{i j_{0}} x_{i} \\
& =\sum_{i=1}^{n 0}\left|x_{i}\right|+\sum_{n>n_{0}} \varepsilon_{i j_{0}} x_{i}
\end{aligned}
$$

thus $\left|\|x\|_{1}-\langle x \mid y\rangle\right|<\varepsilon$ which shows that $\|\cdot\|_{1}$ is selfpolar. It may thus happen 
that a selfpolar norm is not equivalent to any quadratic norm.

That $l^{1}(\mathbf{N})$ admits an indefinite inner product making $\|\cdot\|_{1}$ selfpolar was first shown by Lance in [4]. The inner product defined in equation (2.35) was pointed out to the author by $\mathrm{H}$. Araki.

\section{§3. Quasi-Decomposition Norms}

Let $(\mathscr{H},\langle\cdot \mid \cdot\rangle)$ be an indefinite inner product space allowing a Hilbert space topology which makes $\langle\cdot \mid \cdot\rangle$ jointly continuous and let $(\cdot \mid \cdot)$ be a choice of a Hilbert space structure implementing the topology.

Lemma 3.1. Let $p$ be a quadratic selfpolar norm on $\mathscr{H}$ and let $T$ and $S$ be the operators of Theorem 2.5 and Proposition 2.6.

There is a linear involution $\Gamma$ with domain $R\left(T^{1 / 2}\right)$ such that

$$
\Gamma T^{1 / 2} x=T^{1 / 2} S x, \quad \forall x \in \mathscr{H} .
$$

Proof. Equation (3.1) gives a well-defined linear mapping $\Gamma: R\left(T^{1 / 2}\right)$ $\rightarrow R\left(T^{1 / 2}\right)$. For $x \in \mathscr{H}$ we have $\Gamma^{2} T^{1 / 2} x=\Gamma T^{1 / 2} S x=T^{1 / 2} S S x=T^{1 / 2} x$.

Q.E.D.

The auxiliary operators $T, S$ and $\Gamma$ depend on the choice of the positive definite inner product $(\cdot \mid \cdot)$ which although not unique will be kept fixed throughout this section unless otherwise stated.

Let $p$ be a quadratic selfpolar norm on $\mathscr{H}$. We set

$$
\mathscr{D}=\left\{x \in R\left(T^{1 / 2}\right) \mid S x \in R\left(T^{1 / 2}\right)\right\} .
$$

Lemma 3.2. $D\left(\Gamma^{*}\right)=T^{-1 / 2} \mathscr{D}$.

Proof. For $y \in R\left(T^{1 / 2}\right)$ we have

$$
\begin{aligned}
& \left(S T^{1 / 2} x \mid T^{-1 / 2} y\right)=\left(x \mid T^{1 / 2} S T^{-1 / 2} y\right) \\
& =\left(x \mid \Gamma T^{1 / 2} T^{-1 / 2} y\right)=(x \mid \Gamma y),
\end{aligned}
$$

$\forall x \in \mathscr{H}$,

which proves that $x \in D\left(\Gamma^{*}\right)$ if and only if $S T^{1 / 2} x \in R\left(T^{1 / 2}\right)$, that is if and only if $T^{1 / 2} x \in \mathscr{D}$.

Q.E.D.

Proposition 3.3. Let $p$ be a quadratic selfpolar norm on $(\mathscr{H},\langle\cdot \mid \cdot\rangle)$ and put

$$
\mathscr{H}^{ \pm}=\left\{x \in \mathscr{H} \mid \pm\langle x \mid x\rangle=p(x)^{2}\right\}
$$

Then $\mathscr{H}^{+}$and $\mathscr{H}^{-}$are closed subspaces satisfying 
(i ) $\mathscr{H}^{ \pm}=T^{-1 / 2}\{x \in \mathscr{D} \mid S x= \pm x\}$,

(ii) $\mathscr{H}^{+} \perp \mathscr{H}^{-}$,

(iii) $\mathscr{H}^{+} \oplus \mathscr{H}^{-}=T^{-1 / 2} \mathscr{D}$.

Proof. Let $x=T^{-1 / 2} y$ where $y \in \mathscr{D}$ and $S y= \pm y$. Using Theorem 2.5 (ii) and Proposition 2.6 (ii) we get

$$
\begin{aligned}
\langle x \mid x\rangle & =\left(T^{-1 / 2} y \mid \eta T^{-1 / 2} y\right)=\left(y \mid T^{-1 / 2} \eta T^{-1 / 2} y\right) \\
& =(y \mid S y)= \pm(y \mid y)= \pm\left\|T^{1 / 2} x\right\|^{2}= \pm p(x)^{2} .
\end{aligned}
$$

Suppose conversely that $x \in \mathscr{H}$ and $\langle x \mid x\rangle= \pm p(x)^{2}$, that is $(x \mid \eta x)= \pm\left\|T^{1 / 2} x\right\|^{2}$ or

$$
\left(T^{1 / 2} x \mid S T^{1 / 2} x\right)= \pm\left(T^{1 / 2} x \mid T^{1 / 2} x\right) .
$$

Since $S$ is a unitary we obtain $S T^{1 / 2} x= \pm T^{1 / 2} x$, thus $T^{1 / 2} x \in \mathscr{D}$ which proves (i). It follows from (3.4) that $\mathscr{H}^{+}$and $\mathscr{H}^{-}$are closed and from (i) that $\mathscr{H}^{+}$and $\mathscr{H}^{-}$are subspaces of $\mathscr{H}_{\text {. }}$

Let $x^{ \pm} \in \mathscr{H}^{ \pm} ; x^{ \pm}=T^{-1 / 2} y^{ \pm}$where $y^{ \pm} \in \mathscr{D}$ and $S y^{ \pm}= \pm y^{ \pm}$. Using that $S$ is selfadjoint we get

$$
\left\langle x^{+} \mid x^{-}\right\rangle=\left(T^{-1 / 2} y^{+} \mid \eta T^{-1 / 2} y^{-}\right)=\left(y^{+} \mid S y^{-}\right)=0 .
$$

We notice that $S$ leaves $\mathscr{D}$ invariant, indeed if $z \in \mathscr{D}$ then $S z \in R\left(T^{1 / 2}\right)$ and $S S z=z \in R\left(T^{1 / 2}\right)$. Consequently

$$
\mathscr{D}=\{z \in \mathscr{D} \mid S z=z\}+\{z \in \mathscr{D} \mid S z=-z\} .
$$

This shows that $\mathscr{H}^{+}+\mathscr{H}^{-}=T^{-1 / 2} \mathscr{D}$. Since $p$ is a norm the sum is direct.

$$
\text { Q.E.D. }
$$

Note that the subspaces $\mathscr{H}^{+}$and $\mathscr{H}^{-}$are independent of the choice of the Hilbert space structure $(\cdot \mid \cdot)$.

Theorem 3.4. Let $p$ be a quadratic selfpolar norm and $\mathscr{H}^{ \pm}$the subspaces of Proposition 3.3. We define a linear operator $J$ with domain $D(J)$ $=\mathscr{H}^{+} \oplus \mathscr{H}^{-}$by setting

$$
J\left(x^{+}+x^{-}\right)=x^{+}-x^{-}, \quad x^{+} \in \mathscr{H}^{+}, x^{-} \in \mathscr{H}^{-} .
$$

We have that

(i) $J$ is a closed involution,

(ii) $J=\Gamma^{*}$,

(iii) $J=T^{-1} \eta$, 
(iv) $p(x)=\langle x \mid J x\rangle^{1 / 2}$, for $x \in \mathscr{H}^{+} \oplus \mathscr{H}^{-}$.

Proof. According to Lemma 3.2 and (iii) in Proposition 3.3 we have

$$
D\left(\Gamma^{*}\right)=T^{-1 / 2} \mathscr{D}=\mathscr{H}^{+} \oplus \mathscr{H}^{-}=D(J) .
$$

Let $x \in \mathscr{H}^{+} \oplus \mathscr{H}^{-}, x=x^{+}+x^{-}, x^{+} \in \mathscr{H}^{+}, x^{-} \in \mathscr{H}^{-}$and $y \in D(\Gamma)=R\left(T^{1 / 2}\right)$. Now $x^{ \pm}=T^{-1 / 2} z^{ \pm}$where $z^{ \pm} \in \mathscr{D}$ and $S z^{ \pm}= \pm z^{ \pm}$, and $y=T^{1 / 2} y_{0}, y_{0} \in \mathscr{H}$. We have

$$
\begin{aligned}
(\Gamma y \mid x) & =\left(\Gamma T^{1 / 2} y_{0} \mid x^{+}+x^{-}\right) \\
& =\left(T^{1 / 2} S y_{0} \mid T^{-1 / 2} z^{+}+T^{-1 / 2} z^{-}\right) \\
& =\left(S y_{0} \mid z^{+}+z^{-}\right)=\left(y_{0} \mid z^{+}-z^{-}\right) \\
& =\left(T^{1 / 2} y_{0} \mid T^{-1 / 2} z^{+}-T^{-1 / 2} z^{-}\right) \\
& =\left(y \mid x^{+}-x^{-}\right)=(y \mid J x) .
\end{aligned}
$$

This proves that $J=\Gamma^{*}$, hence $J$ is closed. Furthermore

$$
\begin{aligned}
p(x)^{2} & =\left\|T^{1 / 2} x\right\|^{2}=\left\|T^{1 / 2} x^{+}+T^{1 / 2} x^{-}\right\|^{2} \\
& =\left\|z^{+}+z^{-}\right\|^{2}=\left\|z^{+}\right\|^{2}+\left\|z^{-}\right\|^{2} \\
& =\left\|T^{1 / 2} x^{+}\right\|^{2}+\left\|T^{1 / 2} x^{-}\right\|^{2} \\
& =p\left(x^{+}\right)^{2}+p\left(x^{-}\right)^{2} \\
& =\left\langle x^{+} \mid x^{+}\right\rangle-\left\langle x^{-} \mid x^{-}\right\rangle \\
& =\langle x \mid J x\rangle
\end{aligned}
$$

which shows (iv). In order to prove (iii) we note that

$$
\Gamma=T^{1 / 2} S T^{-1 / 2} .
$$

Since $T^{1 / 2} S$ is bounded we obtain

$$
\begin{aligned}
J & =\Gamma^{*}=T^{-1 / 2}\left(T^{1 / 2} S\right)^{*}=T^{-1 / 2} S T^{1 / 2} \\
& =T^{-1 / 2} T^{-1 / 2} \eta=T^{-1} \eta .
\end{aligned}
$$

Definition 3.5. A semidefinite subspace $\mathscr{K}$ of $(\mathscr{H},\langle\cdot \mid \cdot\rangle)$ is said to be regular if for each $x \in \mathscr{H}$ there is a constant $c(x)>0$ such that

$$
|\langle x \mid y\rangle| \leqq c(x)|\langle y \mid y\rangle|^{1 / 2},
$$

$\forall y \in \mathscr{K}$.

Proposition 3.6. Let $\mathscr{K}$ be a semidefinite regular subspace. Then

(i) $\mathscr{K}$ is definite,

(ii) $\overline{\mathscr{K}}$ is regular,

(iii) $\overline{\mathscr{K}}$ is definite.

Proof. Let $y \in \mathscr{K}$ and assume $\langle y \mid y\rangle=0$. We have

$$
|\langle x \mid y\rangle| \leqq c(x)|\langle y \mid y\rangle|^{1 / 2}=0,
$$


hence $y=0$ which proves (i). The joint continuity of $\langle\cdot \mid \cdot\rangle$ ensures (ii) and $\begin{array}{ll}\text { (iii) follows from (i) and (ii). } & \text { Q.E.D. }\end{array}$

Lemma 3.7. Let $\mathscr{K}$ be a definite regular subspace of $(\mathscr{H},\langle\cdot \mid \cdot\rangle)$. There is a continuous linear mapping $P$ from $\mathscr{H}$ into the intrinsic completion $(\overline{\mathscr{K}}$, $\left.\left.\langle\cdot \mid \cdot\rangle\right|^{1 / 2}\right)$ of $\mathscr{K}$ such that

$$
\begin{aligned}
& \langle x \mid y\rangle=\langle P x \mid y\rangle, \\
& P x=x \text {, } \\
& \forall x \in \mathscr{H}, \forall y \in \mathscr{K}, \\
& \forall x \in \mathscr{K} .
\end{aligned}
$$

Proof. Let $x \in \mathscr{H}$. Since $\mathscr{K}$ is regular the linear form

$$
\mathscr{K} \ni y \longrightarrow\langle x \mid y\rangle
$$

is continuous in the intrinsic topology on $\mathscr{K}$. Hence there is a unique vector $P x$ in the intrinsic completion of $\mathscr{K}$ such that $\langle x \mid y\rangle=\langle P x \mid y\rangle \forall y \in \mathscr{K}$. The uniqueness of the vector $P x$ ensures that the mapping $P: \mathscr{H} \rightarrow\left(\bar{K},|\langle\cdot \mid \cdot\rangle|^{1 / 2}\right)$ is well-defined and linear. Let now $\left(x_{n}\right)_{n \in \boldsymbol{N}}$ be a sequence in $\mathscr{H}$ tending to 0 in the underlying Hilbert space topology on $\mathscr{H}$ and assume $P x_{n} \rightarrow a \in\left(\overline{\mathscr{K}},|\langle\cdot \mid \cdot\rangle|^{1 / 2}\right)$ in the intrinsic topology. Then

$$
\begin{aligned}
\langle a \mid y\rangle & =\lim \left\langle P x_{n} \mid y\right\rangle \\
& =\lim \left\langle x_{n} \mid y\right\rangle=0, \quad \forall y \in \mathscr{K},
\end{aligned}
$$

hence $a=0$. This shows that $P$ is closable. Since the domain of $P$ is $\mathscr{H}$ we conclude that $P$ is closed and continuous. The second statement of the lemma follows from the first.

Q.E.D.

Theorem 3.8. Let $\mathscr{H}^{+}, \mathscr{H}^{-}$be a pair of subspaces of $\mathscr{H}$ such that

(i ) $\langle\cdot \mid \cdot\rangle$ is positive (negative) definite on $\mathscr{H}^{+}\left(\mathscr{H}^{-}\right)$,

(ii) $\left(\mathscr{H}^{+}\right)^{\perp}=\mathscr{H}^{-},\left(\mathscr{H}^{-}\right)^{\perp}=\mathscr{H}^{+}$,

(iii) $\mathscr{H}^{+} \oplus \mathscr{H}^{-}$is dense in $\mathscr{H}$,

(iv) $\mathscr{H}^{+}$and $\mathscr{H}^{-}$are regular.

There is a unique selfpolar quadratic norm $p$ on $\mathscr{H}$ such that

$$
p(x)=\sqrt{\left\langle x^{+} \mid x^{+}\right\rangle-\left\langle x^{-} \mid x^{-}\right\rangle}
$$

for $x \in \mathscr{H}^{+} \oplus \mathscr{H}^{-}, x=x^{+}+x^{-}, x^{+} \in \mathscr{H}^{+}, x^{-} \in \mathscr{H}^{-}$. Furthermore

$$
\mathscr{H}^{ \pm}=\left\{x \in \mathscr{H} \mid \pm\langle x \mid x\rangle=p(x)^{2}\right\} .
$$

Proof. Applying Lemma 3.7 to the subspaces $\mathscr{H}^{+}$and $\mathscr{H}^{-}$we get continuous linear mappings $P^{ \pm}: \mathscr{H} \rightarrow\left(\overline{\mathscr{H}}^{ \pm},|\langle\cdot \mid \cdot\rangle|^{1 / 2}\right)$ such that 


$$
\begin{array}{lr}
\langle x \mid y\rangle=\left\langle P^{ \pm} x \mid y\right\rangle, & \forall x \in \mathscr{H}, \quad \forall y \in \mathscr{H}^{ \pm}, \\
P^{ \pm} x=x, & \forall x \in \mathscr{H}^{ \pm} .
\end{array}
$$

We define

$$
p(x)=\sqrt{\left\langle P^{+} x \mid P^{+} x\right\rangle-\left\langle P^{-} x \mid P^{-} x\right\rangle},
$$

$x \in \mathscr{H}$

and using the continuity of $P^{+}$and $P^{-}$we get

$$
p(x) \leqq\|x\| \sqrt{\left\|P^{+}\right\|_{0}^{2}+\left\|P^{-}\right\|_{0}^{2}}, \quad \forall x \in \mathscr{H},
$$

where $\left\|P^{ \pm}\right\|_{0}$ denotes the norm of $P^{ \pm}$as a linear operator from $\mathscr{H}$ into the intrinsic completion $\left(\overline{\mathscr{H}}^{ \pm},|\langle\cdot \mid \cdot\rangle|^{1 / 2}\right)$. Applying the techniques of the proof of Lemma 2.4 we can easily prove that the restriction of $p$ to $\mathscr{H}^{+} \oplus \mathscr{H}^{-}$is selfpolar. Now in view of (3.19) and condition (iii) we can carry out arguments similar to the statements in equations (2.8), (2.9) and (2.10) and conclude that $p$ is selfpolar on $\mathscr{H}$. The formula (3.15) follows from (3.17) and the definition of $p$. The uniqueness of $p$ follows from (iii) and the continuity of a selfpolar norm given by Proposition 2.2. By equation (3.15) we have

$$
\mathscr{H}^{ \pm} \subseteq\left\{x \in \mathscr{H} \mid \pm\langle x \mid x\rangle=p(x)^{2}\right\} .
$$

Let $T$ and $S$ be the auxiliary operators corresponding to the quadratic selfpolar norm $p$. Using (i) in Proposition 3.3 we get

$$
\mathscr{H}^{ \pm} \subseteq T^{-1 / 2}\{z \in \mathscr{D} \mid S z= \pm z\} .
$$

Let now $z \in \mathscr{D}$ and suppose $S z= \pm z$. It follows from (iii) in Theorem 3.4 that $\eta x=T J x$ for $x \in D(J)$. Consequently

$$
\begin{aligned}
& \left\langle y \mid T^{-1 / 2} z\right\rangle=\left(y \mid \eta T^{-1 / 2} z\right) \\
& =\left(\mp T y \mid T^{-1 / 2} z\right)=\mp\left(T^{1 / 2} y \mid z\right)=0, \quad \forall y \in \mathscr{H}^{\mp} .
\end{aligned}
$$

The last equation follows because $S$ is selfadjoint and $S T^{1 / 2} y=\mp T^{1 / 2} y$. This shows that $T^{-1 / 2} z \in\left(\mathscr{H}^{\mp}\right)^{\perp}=\mathscr{H}^{ \pm}$, thus

$$
\mathscr{H}^{ \pm}=T^{-1 / 2}\{z \in \mathscr{D} \mid S z= \pm z\}
$$

which together with (i) in Proposition 3.3 proves equation (3.16). Q.E.D.

Comparison of equation (3.16) and equation (3.4) shows that the given subspaces $\mathscr{H}^{+}$and $\mathscr{H}^{-}$in Theorem 3.8 coincide with the definite subspaces occuring in Proposition 3.3 corresponding to the quadratic selfpolar norm $p$ given by equation (3.15). We can therefore rewrite equation (3.15) as

$$
p(x)=\langle x \mid J x\rangle^{1 / 2}, \quad \forall x \in \mathscr{H}^{+} \oplus \mathscr{H}^{-},
$$


where $J$ is the closed involution defined in equation (3.7).

Definition 3.9. The quadratic selfpolar norm $p$ constructed in Theorem 3.8 is said to be a quasi-decomposition norm and the pair $\left(\mathscr{H}^{+}, \mathscr{H}^{-}\right)$is called the quasi-decomposition of $\mathscr{H}$ corresponding to $p$.

If $\mathscr{H}^{+} \oplus \mathscr{H}^{-}=\mathscr{H}$ we say that $p$ is a (genuine) decomposition norm and the pair $\left(\mathscr{H}^{+}, \mathscr{H}^{-}\right)$is referred to as the (genuine) decomposition of $\mathscr{H}$ corresponding to $p$. In this case condition (iv) of Theorem 3.8 becomes redundant.

We conclude from (i) in Theorem 3.4 that a quasi-decomposition norm $p$ is a decomposition norm if and only if the involution $J$ is continuous in the underlying Hilbert space topology.

The norm $p$ constructed in Lemma 2.4 is a decomposition norm. If conversely $p$ is an arbitrary decomposition norm and $(\cdot \mid \cdot)$ is a choice of Hilbert space structure, we put $P^{ \pm}=(1 \pm J) / 2$ and

$$
(x \mid y)_{1}=\left(P^{+} x \mid P^{+} y\right)+\left(P^{-} x \mid P^{-} y\right), \quad x, y \in \mathscr{H} .
$$

It is easily verified that $(\cdot \mid \cdot)_{1}$ defines a Hilbert space structure on $\mathscr{H}$ equivalent to $(\cdot \mid \cdot)$ and that $p$ is the norm constructed in Lemma 2.4 relative to this structure.

Corollary 3.10. A complete quadratic selfpolar norm $p$ on $(\mathscr{H},\langle\cdot \mid \cdot\rangle)$ is a decomposition norm.

Proof. We can use $p$ as Hilbert space norm on $\mathscr{H}$. It follows chat $T=1$ and $\mathscr{D}=\mathscr{H}$, hence

$$
\mathscr{H}^{+} \oplus \mathscr{H}^{-}=T^{-1 / 2} \mathscr{D}=\mathscr{H} . \quad \text { Q.E.D. }
$$

Corollary 3.11. Let $p$ be a quadratic selfpolar norm on $(\mathscr{H},\langle\cdot \mid \cdot\rangle)$ and let $(\mathscr{K},\langle\cdot \mid \cdot\rangle)$ denote the $p$-completion of $(\mathscr{H},\langle\cdot \mid \cdot\rangle)$. Then $p$ is a decomposition norm on $(\mathscr{K},\langle\cdot \mid \cdot\rangle)$ and

$$
\mathscr{H}^{ \pm}=\mathscr{H} \cap \mathscr{K}^{ \pm}
$$

where $\mathscr{H}^{+}$and $\mathscr{H}^{-}$are the subspaces defined in equation (3.4) and $\mathscr{K}^{ \pm}$is the decomposition of $\mathscr{K}$ corresponding to $p$.

Proof. It follows from the proof of Proposition 2.2 that $(\mathscr{K},\langle\cdot \mid \cdot\rangle)$ is an indefinite inner product space and that the extension of $p$ to $(\mathscr{K},\langle\cdot \mid \cdot\rangle)$ is quadratic and selfpolar. Thus $p$ is a decomposition norm on $(\mathscr{K},\langle\cdot \mid \cdot\rangle)$ according to Corollary 3.10. Equation (3.27) follows by applying equation 
(3.4) to $p$ on both $(\mathscr{H},\langle\cdot \mid \cdot\rangle)$ and $(\mathscr{K},\langle\cdot \mid \cdot\rangle)$.

Q.E.D.

Theorem 3.12. Let $p$ be a quadratic selfpolar norm on $\mathscr{H}$ and $\Gamma$ the involution defined in Lemma 3.1. Then we have

(i) $p$ is a quasi-decomposition norm if and only if $\Gamma$ is closable,

(ii) $p$ is a decomposition norm if and only if $\Gamma$ is continuous.

Proof. Let $\mathscr{H}^{+}$and $\mathscr{H}^{-}$be the subspaces defined in equation (3.4) and $J$ the involution of Theorem 3.4. If $p$ is a quasi-decomposition norm we obtain from equation (3.16) and (ii) in Theorem 3.4 that $\Gamma^{*}$ is densely defined, thus $\Gamma$ is closable.

Suppose now that $\Gamma$ is closable. We will verify that $\mathscr{H}^{+}$and $\mathscr{H}^{-}$satisfy conditions (i), (ii), (iii) and (iv) of Theorem 3.8 and equation (3.15) with respect to $p$. Condition (i) follows because $p$ is a norm. Since $\Gamma$ is closable $\mathscr{H}^{+} \oplus \mathscr{H}^{-}$ $=D(J)=D\left(\Gamma^{*}\right)$ is dense in $\mathscr{H}$ giving condition (iii). It is already shown in (ii) of Proposition 3.3 that $\mathscr{H}^{+} \perp \mathscr{H}^{-}$. We obtain from (iii) in the same proposition that $T^{-1 / 2} \mathscr{D}=\mathscr{H}^{+} \oplus \mathscr{H}^{-}$and conclude that $\mathscr{D}$ is dense in $\mathscr{H}$.

We first show that $\{z \in \mathscr{D} \mid S z=z\}$ is dense in the nullspace $N(S-1)$. Let $z_{0} \in N(S-1)$ and assume

$$
\left(z_{0} \mid z\right)=0, \quad \forall z \in \mathscr{D}, S z=z
$$

Since $S$ is selfadjoint $\left(z_{0} \mid z\right)=0, \forall z \in \mathscr{D}, S z=-z$. In view of (3.6) we have $\left(z_{0} \mid z\right)=0, \forall z \in \mathscr{D}$ which implies $z_{0}=0$ as $\mathscr{D}$ is dense in $\mathscr{H}$.

Take now $x \in\left(\mathscr{H}^{\top}\right)^{\perp}$. Using (ii) in Theorem 2.5 and (i) in Proposition 3.3 we obtain

$$
\left(T^{-1 / 2} \eta x \mid z\right)=\left\langle x \mid T^{-1 / 2} z\right\rangle=0, \quad \forall z \in \mathscr{D}, S z=z .
$$

From the vanishing of $z_{0} \in N(S-1)$ satisfying (3.28) it follows that $T^{-1 / 2} \eta x$ $\in N(S+1)$, hence

$$
T^{1 / 2} x=S T^{-1 / 2} \eta x=-T^{-1 / 2} \eta x \in N(S+1) .
$$

Consequently $S T^{1 / 2} x=-T^{1 / 2} x$ and $x \in \mathscr{H}^{-}$which proves that $\left(\mathscr{H}^{+}\right)^{\perp}=\mathscr{H}^{-}$. A similar argument shows that $\left(\mathscr{H}^{-}\right)^{\perp}=\mathscr{H}^{+}$, hence condition (ii) is satisfied. To verify condition (iv) take $x \in \mathscr{H}$ and $y \in \mathscr{H}^{ \pm}$. Then

$$
|\langle x \mid y\rangle| \leqq p(x) p(y)=p(x)|\langle y \mid y\rangle|^{1 / 2} .
$$

Finally (iv) in Theorem 3.4 shows that $\mathscr{H}^{+}$and $\mathscr{H}^{-}$satisfy equation (3.15) with respect to $p$, hence $p$ is a quasi-decomposition norm with $\left(\mathscr{H}^{+}, \mathscr{H}^{-}\right)$as 
quasi-decomposition. This proves (i).

Let $p$ be a decomposition norm, then $J$ is continuous and $\Gamma$ is closable. In particular

$$
\bar{\Gamma}=J^{*}
$$

which shows that $\Gamma$ is continuous.

If on the other hand $\Gamma$ is continuous, then so is $J=\Gamma^{*}$. Furthermore $\Gamma$ is closable such that $p$ is a quasi-decomposition norm according to (i). We recall that a quasi-decomposition norm $p$ for which $J$ is continuous is a decomposition norm.

Q.E.D.

It can happen that the involution $J$ corresponding to a quadratic selfpolar norm $p$ is continuous without being densely defined.

Example 3.13. Let $\boldsymbol{Z}_{0}=\boldsymbol{Z} \backslash\{0\}$ and take $\mathscr{H}=l^{2}\left(\boldsymbol{Z}_{0}\right)$. We define

$$
\langle x \mid y\rangle=\sum_{n \in Z_{0}}(\operatorname{sign} n) 2^{-|n|} \bar{x}_{n} y_{n}, \quad x, y \in \mathscr{H},
$$

and observe that $(\mathscr{H},\langle\cdot \mid \cdot\rangle)$ is an indefinite inner product space allowing a Hilbert space topology which makes $\langle\cdot \mid \cdot\rangle$ jointly continuous. For $\lambda \in[1,2]$ and $x \in \mathscr{H}$ we define

$$
p_{\lambda}(x)=\left(\sum_{n \in Z_{0}} 2^{-|n|}\left(\lambda^{|n|}\left|x_{n}\right|^{2}+\sqrt{\lambda^{2|n|}-1} \bar{x}_{n} x_{-n}\right)\right)^{1 / 2} .
$$

A straightforward computation shows that $p_{\lambda}$ is a quasi-decomposition norm with corresponding quasi-decomposition

$$
\mathscr{H}_{\lambda}^{ \pm}=\left\{x \in \mathscr{H} \mid x_{-n}=\mu_{n}^{ \pm} x_{n} \quad \forall n \in \boldsymbol{Z}_{0}\right\}
$$

where

$$
\mu_{n}^{ \pm}=\frac{ \pm \operatorname{sign} n-\lambda^{|n|}}{\sqrt{\lambda^{2|n|}-1}}
$$

The involution $J_{\lambda}$ is given by

$$
\left(J_{\lambda} x\right)_{n}=\operatorname{sign} n\left(\lambda^{|n|} x_{n}+\sqrt{\lambda^{2}|n|}-1 x_{-n}\right),
$$$$
n \in \boldsymbol{Z}_{0},
$$

for $x \in \mathscr{H}_{\lambda}^{+} \oplus \mathscr{H}_{\lambda}$. This shows that $p_{1}$ is a decomposition norm while $p_{\lambda}$ for $\lambda \in] 1,2]$ is not.

If $(x \mid y)=\sum_{n \in Z_{0}} \bar{x}_{n} y_{n}$ is chosen as Hilbert space structure on $\mathscr{H}$ the auxiliary operators $T_{\lambda}, T_{\lambda}^{1 / 2}, S_{\lambda}$ and $\Gamma_{\lambda}$ take the form

$$
\left(T_{\lambda} x\right)_{n}=2^{-|n|}\left(\lambda^{|n|} x_{n}+\sqrt{\lambda^{2|n|}-1} x_{-n}\right),
$$




$$
\begin{aligned}
& \left(T_{\lambda}^{1 / 2} x\right)_{n}=2^{-|n| / 2}\left(\sqrt{\frac{\lambda^{|n|}+1}{2}} x_{n}+\sqrt{\frac{\lambda^{|n|}-1}{2}} x_{-n}\right), \\
& \left(S_{\lambda} x\right)_{n}=(\operatorname{sign} n) x_{n},
\end{aligned}
$$

for $n \in \mathbb{Z}_{0}$ and $x \in \mathscr{H}$,

$$
\left(\Gamma_{\lambda} x\right)_{n}=(\operatorname{sign} n)\left(\lambda^{|n|} x_{n}-\sqrt{\lambda^{2|n|}-1} x_{-n}\right),
$$

for $n \in \boldsymbol{Z}_{0}$ and $x \in R\left(T_{\lambda}^{1 / 2}\right)$.

Let for $r \in[1,2]$ and $N \in \mathbb{N}$ the vector $x(r, N) \in \mathscr{H}$ be given by

$$
x(r, N)_{n}= \begin{cases}0, & n<0, \\ r^{n / 2}, & 0<n \leqq N, \\ 0, & n>N .\end{cases}
$$

It follows that

$$
p_{\lambda}(x(r, N+k)-x(r, N))=\left(\sum_{n=N+1}^{N+k}\left(\frac{\lambda r}{2}\right)^{n}\right)^{1 / 2}
$$

for $N, k \in \mathbb{N}$, hence the sequence $(x(r, N))_{N \in \boldsymbol{N}}$ is convergent to an element in the $p_{\lambda}$-completion of $\mathscr{H}$ if and only if $r<2 / \lambda$. We conclude that the norms $p_{\lambda}$, $\lambda \in[1,2]$ are mutually non-equivalent.

Example 3.14. Let $\mathscr{H}=l^{2}(\mathbf{N})$ and set

$$
\langle x \mid y\rangle=\sum_{n=1}^{\infty} \bar{x}_{n}\left(2^{-n} y_{n}-\frac{12 \cdot 2^{-n / 2}}{\pi^{2} \cdot n} \sum_{k=1}^{\infty} \frac{y_{k}}{k} 2^{-k / 2}\right)
$$

for $x, y \in \mathscr{H}$. It is easily verified that $\langle\cdot \mid \cdot\rangle$ is an indefinite inner product which is jointly continuous in the Hilbert space topology on $\mathscr{H}$. We will prove that the norm

$$
p(x)=\left(\sum_{n=1}^{\infty} 2^{-n}\left|x_{n}\right|^{2}\right)^{1 / 2}, \quad x \in \mathscr{H},
$$

is a quadratic selfpolar norm on $(\mathscr{H},\langle\cdot \mid \cdot\rangle)$ but not a quasi-decomposition norm.

We choose $(x \mid y)=\sum_{n=1}^{\infty} \bar{x}_{n} y_{n}$ as Hilbert space structure on $\mathscr{H}$ and observe that $\langle x \mid y\rangle=(x \mid \eta y), x, y \in \mathscr{H}$, where

$$
(\eta x)_{n}=2^{-n} x_{n}-\frac{12 \cdot 2^{-n / 2}}{\pi^{2} \cdot n} \sum_{k=1}^{\infty} \frac{x_{k}}{k} 2^{-k / 2}
$$

for $n \in \mathbb{N}$ and $x \in \mathscr{H}$. Furthermore $p(x)=\left\|T^{1 / 2} x\right\|, x \in \mathscr{H}$, where

$$
(T x)_{n}=2^{-n} x_{n}, \quad n \in \mathbb{N}, x \in \mathscr{H} .
$$


Let $\xi$ be the vector in $\mathscr{H}$ with components

$$
\xi_{n}=\frac{\sqrt{6}}{n \pi}
$$

We have that

$$
\|\xi\|=\left(\sum_{n=1}^{\infty}\left(\frac{\sqrt{6}}{n \pi}\right)^{2}\right)^{1 / 2}=\frac{\sqrt{6}}{\pi}\left(\sum_{n=1}^{\infty} \frac{1}{n^{2}}\right)^{1 / 2}=1,
$$

thus the operator $P$ defined by

$$
P x=(\xi \mid x) \xi,
$$

$x \in \mathscr{H}$

is a $(\cdot \mid \cdot)$-orthogonal projection and hence $S=1-2 P$ is a selfadjoint unitary. We calculate

$$
\begin{aligned}
\left(T^{-1 / 2} \eta x\right)_{n} & =2^{n / 2}(\eta x)_{n} \\
& =2^{-n / 2} x_{n}-\frac{12}{n \pi^{2}} \sum_{k=1}^{\infty} \frac{x_{k}}{k} 2^{-k / 2} \\
& =\left(T^{1 / 2} x\right)_{n}-2 \zeta_{n} \sum_{k=1}^{\infty} \bar{\xi}_{k}\left(T^{1 / 2} x\right)_{k} \\
& =\left((1-2 P) T^{1 / 2} x\right)_{n} \\
& =\left(S T^{1 / 2} x\right)_{n},
\end{aligned}
$$$$
n \in N, x \in \mathscr{H} \text {. }
$$

It follows from Theorem 2.5 that $p$ is quadratic and selfpolar, furthermore $S$ is the selfadjoint unitary introduced in Proposition 2.6.

The set $\mathscr{D}$ defined in equation (3.2) is dense in $\mathscr{H}$ if $p$ is a quasi-decomposition norm as remarked in the proof of Theorem 3.12. Suppose $x \in \mathscr{D}$, then $x \in R\left(T^{1 / 2}\right)$ and $S x=x-2 P x \in R\left(T^{1 / 2}\right)$, hence $P x \in R\left(T^{1 / 2}\right)$. However $\xi \notin R\left(T^{1 / 2}\right)=l^{2}(\boldsymbol{N}, \mu)$ where $\mu(n)=2^{n}$. We conclude that $P x=0$ and consequently $(\xi \mid x)=0$. This shows that $\mathscr{D}$ is not dense in $\mathscr{H}$ and therefore $p$ is not a quasi-decomposition norm.

\section{§4. Decomposition Equivalence}

Let $(\mathscr{H},\langle\cdot \mid \cdot\rangle)$ be an indefinite inner product space allowing a Hilbert space majorant. If $p$ is a quasi-decomposition norm on $(\mathscr{H},\langle\cdot \mid \cdot\rangle)$ with corresponding quasi-decomposition $\left(\mathscr{H}^{+}, \mathscr{H}^{-}\right)$and $(\cdot \mid \cdot)$ is a choice of Hilbert space structure on $\mathscr{H}$, we introduce a norm $p_{J}$ on $\mathscr{H}^{+} \oplus \mathscr{H}^{-}$by setting

$$
p_{J}(x)=\|J x\|,
$$$$
x \in \mathscr{H}^{+} \oplus \mathscr{H}^{-} .
$$

We note that by (iii) in Theorem 3.4 we have 


$$
\eta x=T J x
$$

$\forall x \in \mathscr{H}^{+} \oplus \mathscr{H}^{-}$

thus the dual norm

$$
\begin{aligned}
p_{J}^{\prime}(x) & =\sup \left\{|\langle x \mid y\rangle| \mid p_{J}(y) \leqq 1, y^{\prime} \in \mathscr{H}^{+} \oplus \mathscr{H}^{-}\right\} \\
& =\sup \left\{|(T x \mid J y)| \mid\|J y\| \leqq 1, y \in \mathscr{H}^{+} \oplus \mathscr{H}^{-}\right\} \\
& =\|T x\|,
\end{aligned}
$$$$
x \in \mathscr{H}
$$

can be defined everywhere in $\mathscr{H}$. It is easily shown that the topology on $\mathscr{H}$ induced by the norm $p_{J}^{\prime}$ is independent of the choice of Hilbert space structure on $\mathscr{H}$ and we will denote this topology by $\tau(p)$.

Proposition 4.1. All decomposition norms on $(\mathscr{H},\langle\cdot \mid \cdot\rangle)$ are equivalent.

Proof. Let $p_{1}$ and $p_{2}$ be decomposition norms on $(\mathscr{H},\langle\cdot \mid \cdot\rangle)$ and let $(\cdot \mid \cdot)$ be a choice of Hilbert space structure on $\mathscr{H}$. The involutions $J_{1}$ and $J_{2}$ corresponding to $p_{1}$ and $p_{2}$ are bounded, hence

$$
\begin{aligned}
p_{J_{2}}(x) & =\left\|J_{2} x\right\|=\left\|J_{2} J_{1} J_{1} x\right\| \\
& \leqq\left\|J_{2} J_{1}\right\| \cdot\left\|J_{1} x\right\|=\left\|J_{2} J_{1}\right\| p_{J_{1}}(x), \quad \forall x \in \mathscr{H} .
\end{aligned}
$$

From equations (1.5) and (4.3) it follows that

$$
\left\|T_{1} x\right\|=p_{J_{1}}^{\prime}(x) \leqq\left\|J_{2} J_{1}\right\| p_{J_{2}}^{\prime}(x)=\left\|J_{2} J_{1}\right\| \cdot\left\|T_{2} x\right\|, \quad \forall x \in \mathscr{H}
$$

which translates into the operator inequality

$$
T_{1}^{2} \leqq\left\|J_{2} J_{1}\right\|^{2} T_{2}^{2} .
$$

Since the square root is operator monotone [2], we obtain

$$
T_{1} \leqq\left\|J_{2} J_{1}\right\| T_{2}
$$

and consequently

$$
\begin{aligned}
p_{1}(x) & =\left\|T_{1}^{1 / 2} x\right\|=\left(x \mid T_{1} x\right)^{1 / 2} \\
& \leqq\left\|J_{2} J_{1}\right\|^{1 / 2}\left(x \mid T_{2} x\right)^{1 / 2} \\
& =\left\|J_{2} J_{1}\right\|^{1 / 2} p_{2}(x), \quad \forall x \in \mathscr{H} .
\end{aligned}
$$

The notion of a decomposition norm can be defined on any indefinite inner product space $(\mathscr{H},\langle\cdot \mid \cdot\rangle)$ and has been studied for some time. Proposition 4.1 is due to G. Wittstock [5], who obtains the result under the weaker condition that $\mathscr{H}$ allows a Banach space topology making $\langle\cdot \mid \cdot\rangle$ jointly continuous. The proof presented here is an adaptation to the language of this article of a proof given in a non-published work of H. Araki, J. Woods and the author. 
Definition 4.2. Let $p_{1}$ and $p_{2}$ be quasi-decomposition norms on ( $\mathscr{H}$, $\langle\cdot \mid \cdot\rangle)$ with corresponding quasi-decompositions $\left(\mathscr{H}_{1}^{+}, \mathscr{H}_{1}^{-}\right)$and $\left(\mathscr{H}_{2}^{+}, \mathscr{H}_{2}^{-}\right)$

We say that $p_{1}$ and $p_{2}$ are decomposition equivalent if

$$
\mathscr{H}_{1}^{+} \oplus \mathscr{H}_{1}^{-}=\mathscr{H}_{2}^{+} \oplus \mathscr{H}_{2}^{-} .
$$

If in this case the densely defined operators $J_{1} J_{2}$ and $J_{2} J_{1}$ are continuous in the underlying Hilbert space topology, we further say that $p_{1}$ and $p_{2}$ are strongly decomposition equivalent.

Due to $J_{i}^{2}=1, i=1,2$ we obtain that both decomposition equivalence and strong decomposition equivalence are equivalence relations in the set of quasidecomposition norms on $(\mathscr{H},\langle\cdot \mid \cdot\rangle)$.

Theorem 4.3. Let $p_{1}$ and $p_{2}$ be quasi-decomposition norms on ( $\mathscr{H}$, $\langle\cdot \mid \cdot\rangle)$. The following implications are valid:

(i) $p_{1}$ and $p_{2}$ are strongly decomposition equivalent.

(ii) $p_{1}$ and $p_{2}$ are decomposition equivalent.

(iii) $p_{1}$ and $p_{2}$ are topologically equivalent.

Proof. That (i) implies (ii) is contained in the definition of strong decomposition equivalence. Suppose now $p_{1}$ and $p_{2}$ are decomposition equivalent. We set

$$
\mathscr{K}=\mathscr{H}_{1}^{+} \oplus \mathscr{H}_{1}^{-}=\mathscr{H}_{2}^{+} \oplus \mathscr{H}_{2}^{-} .
$$

If $(\cdot \mid \cdot)$ is a choice of Hilbert space structure on $\mathscr{H}$ we put

$$
(x \mid y)_{1}=(x \mid y)+\left(J_{1} x \mid J_{1} y\right),
$$

$x, y \in \mathscr{K}$

Since $J_{1}$ is closed with domain $\mathscr{K}$ we conclude that $\mathscr{K}$ equipped with the positive definite inner product $(\cdot \mid \cdot)_{1}$ is a Hilbert space. Furthermore

$$
\begin{aligned}
|\langle x \mid y\rangle| & \leqq\|\eta\| \cdot\|x\| \cdot\|y\| \\
& \leqq\|\eta\| \cdot\|x\|_{1} \cdot\|y\|_{1}, \quad \forall x, y \in \mathscr{K} .
\end{aligned}
$$

By Definition 3.9 we have that $p_{1}$ and $p_{2}$ are decomposition norms on $(\mathscr{K}$, $\langle\cdot \mid \cdot\rangle)$. Since $\mathscr{K}$ allows a Hilbert space majorant $\|\cdot\|_{1}$ we can apply Proposition 4.1 and obtain the desired result.

Q.E.D.

It will be shown in Example 4.6 that neither of the implications in Theorem 4.3 can be reversed.

Proposition 4.4. Let $p_{1}$ and $p_{2}$ be quasi-decomposition norms on 
$(\mathscr{H},\langle\cdot \mid \cdot\rangle)$ and $\left(\mathscr{H}_{1}^{+}, \mathscr{H}_{1}^{-}\right)$respectively $\left(\mathscr{H}_{2}^{+}, \mathscr{H}_{2}^{-}\right)$the corresponding quasidecompositions of $\mathscr{H}$. We define the $\langle\cdot \mid \cdot\rangle$-orthogonal projections

$$
P_{i}^{ \pm}=\frac{1 \pm J_{i}}{2}, \quad i=1,2 .
$$

The norms $p_{1}$ and $p_{2}$ are decomposition equivalent if and only if the restricted mappings

$$
P_{2}^{ \pm}: \mathscr{H}_{1}^{ \pm} \longrightarrow \mathscr{H}_{\frac{1}{2}}^{ \pm} \text {and } P_{1}^{ \pm}: \mathscr{H}_{2}^{ \pm} \longrightarrow \mathscr{H}_{1}^{ \pm}
$$

are everywhere defined and continuous in the underlying Hilbert space topology on $\mathscr{H}$.

Proof. We note that the $\langle\cdot \mid \cdot\rangle$-orthogonal projections $P_{i}^{ \pm}, i=1,2$, are closed with domains $D\left(P_{i}^{ \pm}\right)=D\left(J_{i}\right), i=1,2$. If $p_{1}$ and $p_{2}$ are decomposition equivalent the restricted mappings in equation (4.14) are everywhere defined. Since $\mathscr{H}_{i}^{+}, i=1,2$ are closed it follows that the restricted mappings are continuous. If conversely the mappings of equation (4.14) are everywhere defined (and therefore continuous) we get

$$
D\left(J_{1}\right)=\mathscr{H}_{1}^{+} \oplus \mathscr{H}_{1}^{-} \subset D\left(J_{2}\right)
$$

and symmetrically $D\left(J_{2}\right) \subset D\left(J_{1}\right)$.

Theorem 4.5. Two quasi-decomposition norms $p_{1}$ and $p_{2}$ on $(\mathscr{H},\langle\cdot \mid \cdot\rangle)$ are strongly decomposition equivalent if and only if the topologies $\tau\left(p_{1}\right)$ and $\tau\left(p_{2}\right)$ coincide.

Proof. Let $(\cdot \mid \cdot)$ be a choice of Hilbert space structure on $\mathscr{H}$ and assume $p_{1}$ and $p_{2}$ are strongly decomposition equivalent. We have

$$
\begin{aligned}
p_{J_{2}}(x) & =\left\|J_{2} x\right\|=\left\|J_{2} J_{1} J_{1} x\right\| \leqq\left\|J_{2} J_{1}\right\| \cdot\left\|J_{1} x\right\| \\
& =\left\|J_{2} J_{1}\right\| p_{J_{1}}(x), \quad \forall x \in D\left(J_{1}\right)=D\left(J_{2}\right),
\end{aligned}
$$

and hence according to equation (4.3)

$$
\left\|T_{1} x\right\| \leqq\left\|J_{2} J_{1}\right\| \cdot\left\|T_{2} x\right\|
$$

$\forall x \in \mathscr{H}$

Similarly we prove that

$$
\left\|T_{2} x\right\| \leqq\left\|J_{1} J_{2}\right\| \cdot\left\|T_{1} x\right\|, \quad \forall x \in \mathscr{H},
$$

thus the topologies $\tau\left(p_{1}\right)$ and $\tau\left(p_{2}\right)$ coincide.

If conversely the topologies $\tau\left(p_{1}\right)$ and $\tau\left(p_{2}\right)$ coincide we have 


$$
\frac{1}{c}\left\|T_{1} x\right\| \leqq\left\|T_{2} x\right\| \leqq c\left\|T_{1} x\right\|, \quad \forall x \in \mathscr{H},
$$

for some constant $c>0$. It follows from Lemma 2.7 that $R\left(T_{1}\right)=R\left(T_{2}\right)$. Applying (iii) in Theorem 3.4 we get

$$
\begin{aligned}
D\left(J_{1}\right) & =D\left(T_{1}^{-1} \eta\right)=\left\{x \in \mathscr{H} \mid \eta x \in R\left(T_{1}\right)\right\} \\
& =\left\{x \in \mathscr{H} \mid \eta x \in R\left(T_{2}\right)\right\}=D\left(T_{2}^{-1} \eta\right)=D\left(J_{2}\right)
\end{aligned}
$$

hence $p_{1}$ and $p_{2}$ are decomposition equivalent. From equations (4.3) and (4.19) we now conclude that

$$
\frac{1}{c}\left\|J_{2} x\right\| \leqq\left\|J_{1} x\right\| \leqq c\left\|J_{2} x\right\|, \quad \forall x \in D\left(J_{1}\right)=D\left(J_{2}\right) .
$$

Consequently

$$
\left\|J_{1} J_{2} x\right\| \leqq c\left\|J_{2} J_{2} x\right\|=c\|x\|
$$

and

$$
\left\|J_{2} J_{1} x\right\| \leqq c\left\|J_{1} J_{1} x\right\|=c\|x\|
$$

for $x \in D\left(J_{1}\right)=D\left(J_{2}\right)$.

Q.E.D.

Example 4.6. Let $\boldsymbol{Z}_{0}=\boldsymbol{Z} \backslash\{0\}$ and set $\mathscr{H}=l^{2}\left(\boldsymbol{Z}_{0}, \mu\right)$ where

$$
\mu(n)= \begin{cases}1, & n<0, \\ n, & n>0 .\end{cases}
$$

We define $\langle x \mid y\rangle=\sum_{n \in Z_{0}}(\operatorname{sign} n) \bar{x}_{n} y_{n}, x, y \in \mathscr{H}$ and note that $\langle\cdot \mid \cdot\rangle$ is an indefinite inner product on $\mathscr{H}$ which is jointly continuous in the Hilbert space topology on $\mathscr{H}$. The norms

$$
\begin{array}{ll}
p_{1}(x)=\left(\sum_{n \in Z_{0}}\left|x_{n}\right|^{2}\right)^{1 / 2}, & x \in \mathscr{H}, \\
p_{2}(x)=\left(\sum_{n \in \mathbb{Z}_{0}} \sqrt{2}\left|x_{n}\right|^{2}+\bar{x}_{n} x_{-n}\right)^{1 / 2}, & x \in \mathscr{H}, \\
p_{3}(x)=\left(\sum_{n \in Z_{0}} 2\left|x_{n}\right|^{2}+\sqrt{3} \bar{x}_{n} x_{-n}\right)^{1 / 2}, & x \in \mathscr{H},
\end{array}
$$

are quasi-decomposition norms on $(\mathscr{H},\langle\cdot \mid \cdot\rangle)$ with corresponding involutions

$$
\begin{array}{ll}
\left(J_{1} x\right)_{n}=(\operatorname{sign} n) x_{n}, & n \in \mathbb{Z}_{0}, \\
\left(J_{2} x\right)_{n}=(\operatorname{sign} n)\left(\sqrt{2} x_{n}+x_{-n}\right), & n \in \mathbb{Z}_{0}, \\
\left(J_{3} x\right)_{n}=(\operatorname{sign} n)\left(2 x_{n}+\sqrt{3} x_{-n}\right), & n \in \mathbb{Z}_{0} .
\end{array}
$$

We observe that $J_{1}$ is continuous while $J_{2}$ is not, hence $p_{1}$ and $p_{2}$ are not decomposition equivalent. However, if we extend $\langle\cdot \mid \cdot\rangle, p_{1}$ and $p_{2}$ to the Hilbert 
space $l^{2}\left(\mathbb{Z}_{0}\right)$ the involutions $J_{1}$ and $J_{2}$ become both continuous. It is easily verified that Proposition 4.1 can be applied and it follows that $p_{1}$ and $p_{2}$ are topologically equivalent. The quasi-decompositions corresponding to $p_{2}$ and $p_{3}$ are given by

$$
\begin{aligned}
& \mathscr{H}_{2}^{ \pm}=\left\{x \in \mathscr{H} \mid x_{-n}=\mu_{n}^{ \pm} x_{n}\right\}, \\
& \mathscr{H}_{3}^{ \pm}=\left\{x \in \mathscr{H} \mid x_{-n}=v_{n}^{ \pm} x_{n}\right\}
\end{aligned}
$$

where

$$
\begin{array}{ll}
\mu_{n}^{ \pm}= \pm(\operatorname{sign} n)-\sqrt{2}, & n \in \mathbb{Z}_{0} \\
v_{n}^{ \pm}=-\frac{1}{\sqrt{3}}( \pm(\operatorname{sign} n)-2), & n \in \mathbb{Z}_{0} .
\end{array}
$$

Either by directly showing that

$$
\mathscr{H}_{2}^{+} \oplus \mathscr{H}_{2}^{-}=\mathscr{H}_{3}^{+} \oplus \mathscr{H}_{3}^{-}=l^{2}\left(\mathbb{Z}_{0}, v\right)
$$

where $v(n)=|n|, n \in \mathbb{Z}_{0}$ or by applying Proposition 4.4 we get that $p_{2}$ and $p_{3}$ are decomposition equivalent. The operator $J_{2} J_{3}$ is given by

$$
\left(J_{2} J_{3} x\right)_{n}=(2 \sqrt{2}-\sqrt{3}) x_{n}+(\sqrt{6}-2) x_{-n}, \quad n \in \mathbb{Z}_{0},
$$

and is not continuous in the Hilbert space topology on $\mathscr{H}$. Thus $p_{2}$ and $p_{3}$ are not strongly decomposition equivalent.

\section{Acknowledgement}

The author would like to thank Professor Araki for encouragement and helpful suggestions during the preparation of this article.

\section{References}

[1] Aronszajn, N., Quadratic forms on vector spaces, Proc. Internat. Sympos. Linear Spaces, Jerusalem, 1961, 29-87.

[2] Bendat, J. and Sherman, S., Monotone and convex operator functions, Trans. Amer. Math. Soc., 79 (1955), 58-71.

[ 3 ] Bognár, J., Indefinite Inner Product Spaces, Springer Verlag, Berlin, 1974.

[4] Lance, E. C., Quadratic forms on Banach spaces, Proc. Lon. Math. Soc., 25 (1972), 341-357.

[5] Wittstock, G., Über Zerlegungsmajoranten indefiniter Metriken, Math. Zeitschr., 91 (1966), 421-430. 
